\title{
DETECTION OF METASTATIC BREAST CARCINOMA CELLS IN A BONE MARROW SAMPLE BY FLOW CYTOMETRY: CASE REPORT
}

\author{
Daniella Serafin Couto Vieira¹, Manoela Lira Reis, Sandro Wopereis ${ }^{1}$, Maria Claudia Santos Da Silva² \\ ${ }^{1}$ Hospital Universitário Professor Polydoro Ernani de São Thiago - Florianópolis (SC), Brazil. \\ ²Departamento de Analises Clinicas, Universidade Federal de Santa Catarina - Florianópolis (SC), Brazil.
}

Introduction: Breast cancer is a global public health issue due to its high mortality. Despite the multidisciplinary approaches and strategies to reduce mortality, many women are diagnosed in advanced stages with metastases, compromising the chances of cure. Objective: To report the case of a 28-year-old woman, G3, with a history of postpartum depression, on treatment for post-breastfeeding mastitis, with lumbar pain radiating to the chest and lower limbs for 5 months, progressing to epistaxis, hair loss, lymphadenopathy, dyspnea, lower limb petechiae, weight loss of $20 \mathrm{~kg}$, and bicytopenia in the prior 2 months. She was admitted to the emergency department with fever and night sweats and was diagnosed with metastatic breast cancer based on bone marrow (BM) analysis by flow cytometry (FC). Method: BM biopsy (BMB) was performed and evaluated by FC immunophenotyping using the antibodies anti-CD3, anti-CD4, anti-CD8, anti-CD19, anti-CD56, anti-CD34, anti-CD45, and anti-HER2. The streptavidin-biotin-peroxidase method was adopted for the phenotypic evaluation by immunohistochemistry (IHC). Results: Clinical findings and her history favored the hypothesis of lymphoproliferative neoplasm. BM samples were collected for immunophenotyping and BMB for histological study and IHC. The initial aspirate analysis by FC identified $0.90 \%$ of non-hematological cells, with a positive expression for the antibody anti-HER2, suggesting epithelial neoplasm, which directed the investigation toward solid tumor with unknown primary site. The presence of this cellular component guided the IHC panel of BMB. Cells positive for CKPOOL, CK7, E-cadherin, estrogen receptor, progesterone receptor, HER2, GCDFP15, and mammaglobin were identified, indicating immunophenotype of metastatic breast disease in the BM. Later, a nodule was clinically detected in the right breast, showing a pattern consistent with that found in the BM sample, confirming the metastatic breast carcinoma. Conclusions: FC has proven to be a methodology of great clinical importance. Its routine laboratory application in the diagnosis of solid tumors could become a useful tool, providing agility and increasing diagnostic coverage. 